\title{
K - Domination Number of Butterfly Graphs
}

\author{
P. Vijaya Jyothi ${ }^{1}$, Indrani Kelkar ${ }^{2}$, B. Maheswari ${ }^{3}$ \\ 1(Department of Mathematics, NBKR Institute of Science and Technology, Vidyanagar, Nellore, India) \\ 2(Department of Mathematics, Acharya Institute of Technology, Bangalore, India) \\ 3(Department of Mathematics, Sri Padmavati Mahila Viswavidyalayam, Tirupati, India)
}

\begin{abstract}
Butterfly graphs and domination are very important ideas in computer architecture and communication techniques. We present results about one important domination parameter k-domination Number for Butterfly Graph. We find the relation between domination number and k-domination number for $\mathrm{BF}(\mathrm{n})$. In this paper we present results about k-domination number of Butterfly Graphs $\mathrm{BF}(\mathrm{n})$. We show that domination number and k-domination number of butterfly graphs $\mathrm{BF}(\mathrm{n})$ are related to each other as $\gamma_{\mathrm{k}}(\mathrm{BF}(\mathrm{n}))$ $>\gamma(\mathrm{BF}(\mathrm{n}))$ except for $\mathrm{k}=2$ and $\mathrm{n}=6$.
\end{abstract}

Keywords: Butterfly graph, domination number, k-domination number 2000 Mathematics Subject Classification: 05 C69

\section{INTRODUCTION}

Butterfly Networks are interconnection networks which form the back bone of distributed memory parallel architecture. They have very good symmetry in structure and are regular graphs. One of the current interests of researchers is Butterfly graphs, because they are studied as a topology of parallel machine architecture. The vertices model processors and the edges represent communication links between processors.

The vertex set $\mathrm{V}$ of $\mathrm{BF}(\mathrm{n})$ is the set of ordered pairs $(\alpha ; \mathrm{v})$ where $\alpha \in\{0,1,2, \ldots . . \mathrm{n}-1\}$ and $\mathrm{v}=\mathrm{x}_{\mathrm{n}-1} \mathrm{x}_{\mathrm{n}-2} \ldots \ldots$. $\mathrm{x}_{1} \mathrm{x}_{0}$ is a binary string of length $\mathrm{n}$ where $\mathrm{x}_{\mathrm{i}}=0$ or 1 . There is an edge from a vertex $(\alpha ; \mathrm{v})$ to a vertex $\left(\alpha^{\prime} ; \mathrm{v}^{\prime}\right)$ where $\alpha^{\prime} \equiv \alpha+1(\bmod n)$ and $\mathrm{x}_{\mathrm{j}}=\mathrm{x}_{\mathrm{j}}{ }^{\prime} \forall \mathrm{j} \neq \alpha^{\prime}$. A butterfly graph $\mathrm{BF}(\mathrm{n})$ is an $\mathrm{n}$ - partite graph with $\mathrm{n}$ levels. Each level $L_{k}$ for $k=0,1, \ldots, n-1$ has $2^{n}$ vertices and $L_{k}=\left\{(k ; v) / v=x_{n-1} x_{n-2} \ldots \ldots x_{1} x_{0}, x_{i}=0\right.$ or 1$\}$. Using decimal representation of the binary string we can write $\mathrm{L}_{\mathrm{k}}=\left\{(\mathrm{k} ; \mathrm{m}) /\right.$ where $\mathrm{k}=0,1,2, \ldots . \mathrm{n}-1$ and $\mathrm{m}=\sum \mathrm{x}_{\mathrm{j}} 2^{\mathrm{j}}$ $, \mathrm{j}=0,1, \ldots . \mathrm{n}-1)\}$.

\section{MAIN RESULTS}

The concept of domination in graph theory was formalized by Berge [2] and Ore [9]. A well known upper bound for the domination number of a graph was given by Ore [9].

A subset $\mathrm{D}$ of $\mathrm{G}$ is called a $\mathrm{k}$-dominating set of $\mathrm{G}$ if every vertex in $\mathrm{V} \backslash \mathrm{D}$ is dominated by at least $\mathrm{k}$ vertices in D. Cardinality of a minimum k-dominating set is called the k-domination number of $G$ and is denoted by $\gamma_{k}(G)$. In this paper we present results on the k-domination number of butterfly graph $\mathrm{BF}(\mathrm{n})$. $\mathrm{BF}(\mathrm{n})$ is a 4-regular graph, so k-domination is possible only for $\mathrm{k}<5$ in $\mathrm{BF}(\mathrm{n})$. The result for $\mathrm{k}=2$ is proved in [ 10 ], here we present the proof for $\mathrm{k}=3,4$.

Theorem 1 : The 3-domination number of $\mathrm{BF}(\mathrm{n})$ for $\mathrm{n}>1$ is

$$
\begin{aligned}
\gamma_{3}(\mathrm{BF}(\mathrm{n})) & =\mathrm{n} 2^{\mathrm{n}-1} \text { if } \mathrm{n} \leq 4 \\
& =\left\lceil\frac{n}{2}\right\rceil 2^{n} \quad \text { if } \mathrm{n}=5 \mathrm{k} \quad \mathrm{k}=1,2,3, \ldots .
\end{aligned}
$$

Proof: We give construction of a k-dominating set of $\mathrm{BF}(\mathrm{n})$ for $\mathrm{n}=2,3,4,5$ first and then for $\mathrm{n}>5 \mathrm{k}$ dominating sets are found using special Recursive Construction.

Case (i) $\mathbf{n}=\mathbf{2}$

Consider the butterfly graph $\mathrm{BF}(2)$. In $\mathrm{BF}(2)$ every vertex in one level is adjacent to 3 vertices in the other level. Let $\mathrm{D}$ denote a 3-dominating set of $\mathrm{BF}(2)$. For a vertex to be 3-dominated, at least 3 vertices adjacent with that vertex are needed to be in the 3 -dominating set. So $|\mathrm{D}| \geq 3$.

Let $v=(1, t)$ be a vertex from $L_{1}, v$ has three vertices adjacent in $L_{0}$. To 3-dominate $v$, all these vertices must be included into $\mathrm{D}$. Now there is one vertex left in $\mathrm{L}_{0}$ including it in $\mathrm{D}$ makes each vertex of $\mathrm{L}_{1}$ to be 3-dominated giving $|D|=4$, This is the minimum cardinality as any other 3-dominating set will include vertices from two levels and so will have more than four vertices.

So $\gamma_{3}(\operatorname{BF}(2))=4=2 \times 2^{2-1}=n \times 2^{n-1}$. 
Case (ii) $\mathbf{n}=\mathbf{3}$.

Consider butterfly graph $\mathrm{BF}(3)$. We know that $\mathrm{BF}(3)$ has three levels $\mathrm{L}_{0}, \mathrm{~L}_{1}$ and $\mathrm{L}_{2}$ with 8 vertices each and every vertex in a level $L_{k}$ is adjacent to two vertices in the preceding level $L_{k-1}$ and two vertices in the succeeding level $\mathrm{L}_{\mathrm{k}+1}$. So for 3-domination of a vertex, two vertices from one level and one vertex from other level must be selected into D

Let $\mathrm{D}$ denote a 3-dominating set of $\mathrm{BF}(3)$. Consider a vertex from level $\mathrm{L}_{1}$ $\left(1 ; \mathrm{m}_{1}\right)$ in the left copy of $\mathrm{BF}(3)$, that is $\mathrm{m}_{1} \varepsilon\{0,1,2,3\}$. To 3-dominate this vertex, three adjacent vertices of (1; $\left.\mathrm{m}_{1}\right)$ must be included into $\mathrm{D}$. Vertex $\left(1 ; \mathrm{m}_{1}\right)$ is adjacent to two vertices in $\mathrm{L}_{0}, \quad\left(0 ; \mathrm{m}_{1}\right),\left(0 ; \mathrm{m}_{2}\right)$, with $\mathrm{m}_{1} \neq \mathrm{m}_{2}$ and $\left|\mathrm{m}_{1}-\mathrm{m}_{2}\right|=2$, which belong to $\mathrm{K}_{2,2}$ between $\mathrm{L}_{0}-\mathrm{L}_{1}$ and two vertices in $\mathrm{L}_{2}$ viz. $\left(2 ; \mathrm{m}_{1}\right),\left(2 ; \mathrm{s}_{1}\right)$, with $\mathrm{m}_{1}$ $\neq \mathrm{s}_{1}$ and $\left|\mathrm{m}_{1}-\mathrm{s}_{1}\right|=2^{2}$ where $\mathrm{s}_{1} \varepsilon\{4,5,6,7\}$, which belong to a $\mathrm{K}_{2,2}$ between $\mathrm{L}_{1}$ and $\mathrm{L}_{2}$.

Without loss of generality, take two vertices from $L_{0}$ and one vertex from $L_{2}$ into $D$ giving $D=\left\{\left(0 ; m_{1}\right),\left(0 ; m_{2}\right)\right.$, $\left.\left(2 ; \mathrm{s}_{1}\right)\right\}$, where $\mathrm{s}_{1}=\mathrm{m}_{1}$ or $\left|\mathrm{m}_{1}-\mathrm{s}_{1}\right|=2^{2}$. Vertices of D 3-dominate $\left(1 ; \mathrm{m}_{1}\right)$. Next Consider vertex $\left(1 ; \mathrm{m}_{2}\right)$ which is dominated by $\left(0 ; \mathrm{m}_{1}\right)$ and $\left(0 ; \mathrm{m}_{2}\right)$. To 3 -dominate this vertex, one of its adjacent vertices $\left(2 ; \mathrm{s}_{2}\right)$ with $\mathrm{s}_{2}$ $=\mathrm{m}_{2}$ or $\left|\mathrm{m}_{2}-\mathrm{s}_{2}\right|=2^{2}$ from $\mathrm{L}_{2}$ is to be included where $\mathrm{s}_{1} \neq \mathrm{s}_{2}$.

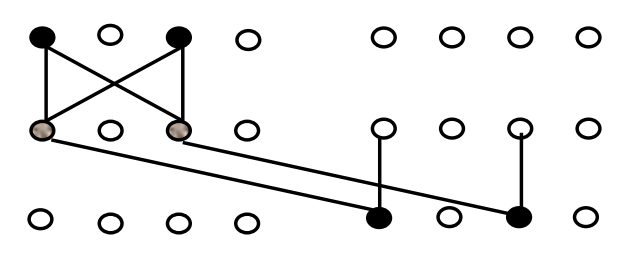

Figure 1

The vertices $\left\{\left(0 ; \mathrm{m}_{1}\right),\left(0 ; \mathrm{m}_{2}\right),\left(2 ; \mathrm{s}_{1}\right),\left(2 ; \mathrm{s}_{2}\right)\right\}$ can 3-dominate only two vertices $\left(1, \mathrm{~m}_{1}\right)$ and $\left(1, \mathrm{~m}_{2}\right)$. None of these four vertices are adjacent to other two vertices from left copy of $\mathrm{L}_{1}$. As shown in figure 1 , to dominate $(1 ; 0)$ and $(1 ; 2)$ we need four vertices $(0 ; 0),(0 ; 2),(2 ; 4),(2 ; 6)$. So to 3 -dominate remaining vertices of level $\mathrm{L}_{1}$ in the left copy we need to include four more vertices to $\mathrm{D}$.

Giving $\mathrm{D}=\left\{\left(0 ; \mathrm{m}_{1}\right),\left(0 ; \mathrm{m}_{2}\right),\left(2 ; \mathrm{s}_{1}\right),\left(2 ; \mathrm{s}_{2}\right),\left(0 ; \mathrm{m}_{3}\right),\left(0 ; \mathrm{m}_{4}\right)\left(2 ; \mathrm{s}_{3}\right),\left(2 ; \mathrm{s}_{4}\right)\right\}$

Now the vertices of $\mathrm{L}_{1}$ in the left copy of $\mathrm{BF}(3)$ are 3 -dominated by the vertices in D. Similarly to 3 dominate vertices of $\mathrm{L}_{1}$ in the right copy, 4 remaining vertices each from levels $\mathrm{L}_{0}$ and $\mathrm{L}_{2}$ need to be included to D. But the vertices of D 2-dominate remaining vertices from $\mathrm{L}_{0}$ and $\mathrm{L}_{2}$ so if we include $\left(1 ; \mathrm{s}_{1}\right),\left(1 ; \mathrm{s}_{2}\right),\left(1 ; \mathrm{s}_{3}\right),(1 ;$ $\mathrm{s}_{4}$ ) into $\mathrm{D}$ then all the vertices of VVD are 3-dominated. Therefore the minimum cardinality of a 3-dominating set is 12

Two possible minimal 3-dominating sets of $\mathrm{BF}(3)$ are

$\mathrm{D}_{1}=\{(0 ; 0),(0 ; 1),(0 ; 2),(0 ; 3),(1 ; 4),(1 ; 5),(1 ; 6),(1 ; 7),(2 ; 4),(2 ; 5),(2 ; 6),(2 ; 7)\}$

$D_{2}=V \backslash D_{1}$, Complement of $D_{1}$. Hence $\gamma_{3}(B F(3))=12=3 \times 2^{3-1}=n \times 2^{n-1}$.

\section{Case (iii) $n=4$}

Consider $\mathrm{BF}(4)$. As in case (ii), to 3 -dominate vertices in level $\mathrm{L}_{1}$ we select 16 vertices from $\mathrm{L}_{0}$ and 8 vertices from $\mathrm{L}_{2}$. These vertices 3-dominate all vertices of level $\mathrm{L}_{3}$ also.

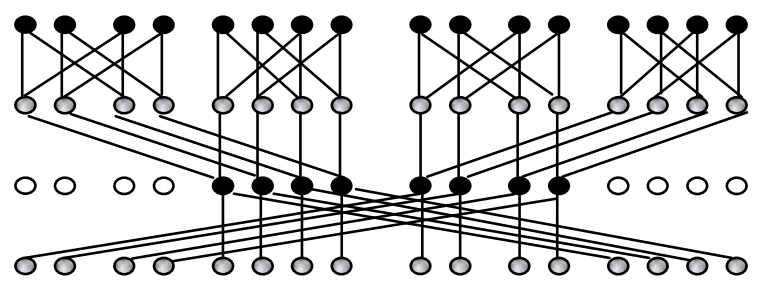

Figure 2

Now to 3-dominate remaining vertices of $\mathrm{L}_{2}$ we have two choices, either select vertices from two levels $\mathrm{L}_{1}$ and $\mathrm{L}_{3}$ or include these 8 vertices into 3-dominating set. For minimal number of vertices in 3-dominating set we include these 8 vertices of $L_{3}$ into 3-dominating set. Therefore all vertices from $L_{1}$ and $L_{3}$ form a 3dominating set of $\mathrm{BF}(4)$. Similarly set of all vertices from $\mathrm{L}_{0}$ and $\mathrm{L}_{2}$ form another 3-dominating set of $\mathrm{BF}(4)$ of same cardinality $2 \times 2^{4}$.

Therefore $\gamma_{3}(B F(4))=2 \times 2^{4}=4 \times 2^{4-1}=n 2^{n-1}$ 
Case (iv) $\mathbf{n}=\mathbf{5}$.

Consider the butterfly graph $\mathrm{BF}(5)$. By the Recursive Construction given in [8], $\mathrm{BF}(5)$ has 2 copies of $\mathrm{BF}(4)$ and a new level $\mathrm{L}_{4}$ with $2^{5}$ vertices. Let $\mathrm{D}_{1}$ and $\mathrm{D}_{2}$ denote 3-dominating sets in the left and right copy of $\mathrm{BF}(4)$ respectively. Since $\gamma_{3}(\mathrm{BF}(4))=32$, select 32 vertices from left copy of $\mathrm{BF}(5)$ into $\mathrm{D}_{1}$ and 32 vertices from right copy of $B F(5)$ into $D_{2}$. Let $D_{1}=\{(0 ; i),(2 ; i) / i=0$ to15 $\}$. Then all the vertices in left copy of $L_{1}$ are 3-dominated by the vertices in $D_{1}$ and the vertices $(3 ; i), \quad i=0 . . .15$ are 2 -dominated. To 3 -dominate these vertices there are two choices. One choice is to select all vertices of $\mathrm{L}_{4}$ and another choice is to select all vertices of $\mathrm{L}_{3}$. If the vertices of $\mathrm{L}_{4}$ are selected, then these vertices will 1-dominate the vertices of $\mathrm{L}_{3}$ which are already 2-dominated. So they will be 3-dominated. Similar is the case with the selection of vertices of $\mathrm{L}_{3}$. Hence the vertices of $L_{4}$ are taken into $D_{1}$ so that $D_{1}=\left\{\left(0 ; m_{i}\right),\left(2 ; m_{i}\right),\left(4 ; m_{i}\right) / i=0.15\right\}$ and $\left|D_{1}\right|=3.2^{4}$.

Using the symmetry of butterfly graphs, mirror image compliment set of $\mathrm{D}_{1}$ in the right copy of $\mathrm{BF}(5)$ will 3dominate all vertices in that copy and it is given by

$\mathrm{D}_{2}=\left\{\left(0 ; \mathrm{m}_{\mathrm{i}}\right),\left(2 ; \mathrm{m}_{\mathrm{i}}\right),\left(4 ; \mathrm{m}_{\mathrm{i}}\right) / \mathrm{i}=16 \ldots . .31\right\}$.

Hence $\mathrm{D}=\mathrm{D}_{1} \cup \mathrm{D}_{2}$ becomes a 3-dominating set of $\mathrm{BF}(5)$ with $|\mathrm{D}|=3.2^{5}=96$.

We claim that $\mathrm{D}$ is a minimum 3-dominating set of $\mathrm{BF}(5)$. If possible let there be a 3-dominating set $\mathrm{S}$ such that $|\mathrm{S}|<\mid \mathrm{D}$. As the domination number of $\mathrm{BF}(\mathrm{n})$ is even, due to the symmetry of the graph, $|\mathrm{S}|$ is even. Now $|\mathrm{S}|<|\mathrm{D}|$ implies $|\mathrm{S}|=94$ or $|\mathrm{S}|<94$. Suppose $|\mathrm{S}|=94$. Again by symmetry, 47 vertices in the left copy and 47 vertices in the right copy are to be selected. But this is not a choice as domination number is even.

Suppose 46 vertices in the left copy and 48 vertices in the right copy are selected. Then as above 48 vertices will 3-dominate all vertices in the right copy of $\mathrm{BF}(3)$. Out of 46 vertices in the left copy, as $\gamma_{3}(\mathrm{BF}(4))=$ $2^{5}$, balance 14 vertices may 2-dominate (it is also not possible) the remaining 16 vertices in $\mathrm{BF}(5)$, but can not 3 -dominate, because to 3 -dominate a vertex we require at least 3 vertices. Similar is the case if $|\mathrm{S}|<94$. Hence $\mathrm{D}$ is a minimum 3-dominating set of $\mathrm{BF}(5)$. Thus $\gamma_{3}(\mathrm{BF}(5))=3.2^{5}==\left\lceil\frac{n}{2}\right\rceil 2^{n}$

One such selection of 3-dominating set for $\mathrm{BF}(5)$ is illustrated in the following figure.

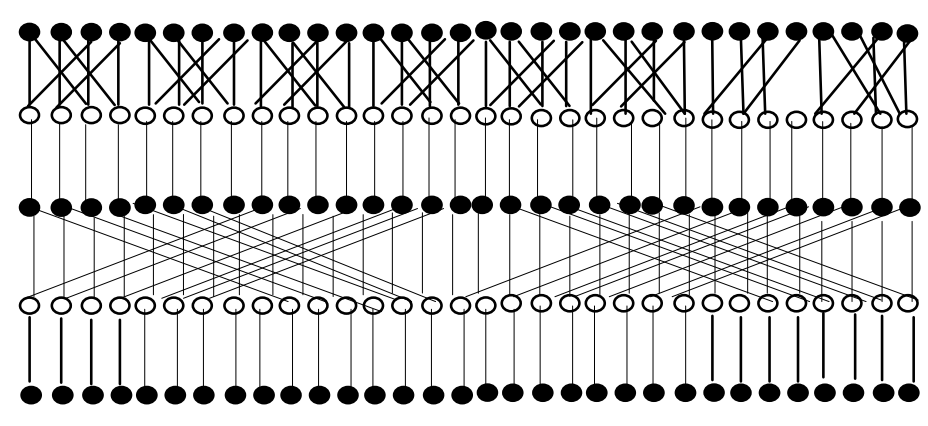

Figure 3

Case (v) $\mathbf{n}=5 \mathrm{k}$

The result is proved by using the Principle of Mathematical Induction on $\mathrm{k}$.

Step 1: Let $\mathrm{k}=1$. So $\mathrm{n}=5$. From case (iv) the result is true for $\mathrm{BF}(5)$.

Step 2: Let us assume that the result is true for $k=t$. Then the result is proved for $k=t+1$. Consider the graph $\mathrm{BF}(5(\mathrm{t}+1))$. From Recursive Construction $2[8], \mathrm{BF}(5 \mathrm{t}+5)$ is decomposed into $2^{5}$ copies of $\mathrm{BF}(5 \mathrm{t})$ without wings and the last 5 levels form a pattern of $\mathrm{BF}(5)$ with $2^{5}$ vertex groups where each vertex group has $2^{5 t}$ vertices.

From the induction hypothesis the result is true for $\mathrm{BF}(5 \mathrm{t})$. So consider a 3-dominating set $\mathrm{S}_{\mathrm{i}}$ of cardinality $\left[\frac{5 t}{2}\right] 2^{5 t}$ for each copy of $\mathrm{BF}(5 t)$. In $\mathrm{BF}(5 t+5)$ the last 5 levels form a pattern of $\mathrm{BF}(5)$, which is isomorphic to $\mathrm{BF}(5)$ without wings. The choice of vertices into a 3-dominating set for $\mathrm{BF}(5)$, is from levels $\mathrm{L}_{0}$, $\mathrm{L}_{2}$ and $\mathrm{L}_{4}$. Hence this result obtained for the graph $\mathrm{BF}(5)$ can be extended to a pattern of $\mathrm{BF}(5)$.

Thus a 3-dominating set $\mathrm{D}_{1}$ is obtained with 32 vertex groups (recall that each vertex group is treated as one vertex) in $\mathrm{L}_{0}, \mathrm{~L}_{2}$ and $\mathrm{L}_{4}$ of the last 5 levels, where each vertex group has $2^{5 \mathrm{t}}$ vertices.

Suppose a 3-dominating set of cardinality less than $32 \times 3 \times 2^{5 t}$ for the pattern of $\operatorname{BF}(5)$ is obtained. Since the pattern of $\mathrm{BF}(5)$ is isomorphic to the graph $\mathrm{BF}(5)$ without wings, this assumption gives the existence of a 3dominating set of cardinality less than $3.2^{5}$ for $\mathrm{BF}(5)$, which is a contradiction as $\gamma_{3}(\mathrm{BF}(5))=3 \times 2^{5}$.

Hence $\mathrm{D}_{1}$ is a minimum 3-dominating set for a pattern of $\mathrm{BF}(5)$. Let $D=\bigcup_{i=1}^{32} S_{i} \cup D_{1}$. 
As $S_{i}$ are minimum 3-dominating sets of disjoint copies of $B F(5 t)$ between levels $L_{0}$ to $L_{5 t-1}$ and $D_{1}$ is a minimum 3-dominating set for pattern of $\mathrm{BF}(5)$ between levels $\mathrm{L}_{5 \mathrm{t}}$ to $\mathrm{L}_{5 \mathrm{t}+4}$, it follows that $\mathrm{D}$ is a disjoint union of minimum 3-dominating sets.

Hence $\mathrm{D}$ is a minimum 3-dominating set of $\mathrm{BF}(5 \mathrm{t}+5)$ whose cardinality is

$|\mathrm{D}|=32 .\left\lceil\frac{5 t}{2}\right\rceil 2^{5 t}+32.3 .2^{5 \mathrm{t}}$.

$$
\begin{gathered}
=\left\lceil\frac{5 t}{2}\right\rceil \cdot 2^{5 t+5}+\left\lceil\frac{5}{2}\right\rceil 2^{5 t+5} . \\
=\left\lceil\frac{5(t+1)}{2}\right\rceil 2^{5(t+1)} .
\end{gathered}
$$

So the result is true for $\mathrm{k}=\mathrm{t}+1$.

Hence by the Principle of Mathematical Induction the result is true for all positive integers k.

Thus $\gamma_{3}(\mathrm{BF}(5 \mathrm{k}))=\left\lceil\frac{5 k}{2}\right\rceil 2^{5 k}=\left\lceil\frac{n}{2}\right\rceil 2^{n}$

Corollary 1: $\gamma_{3}(\mathrm{BF}(5 \mathrm{k}+1))=\left\lceil\frac{5 k+1}{2}\right\rceil 2^{5 k}=\left\lceil\frac{n}{2}\right\rceil 2^{n}$

Proof : In $\mathrm{BF}(5 \mathrm{k}+1)$, from each of two consecutive levels we need to include vertices from one level into 3dominating set for example $\mathrm{L}_{0}, \mathrm{~L}_{2}, \mathrm{~L}_{4}, \ldots \ldots . . \mathrm{L}_{\mathrm{n}-2}$ together 3-dominate all vertices of the other levels giving minimal 3-dominating set with cardinality $\left[\frac{n}{2}\right] 2^{n}$. One such selection of 3-dominating set for $\mathrm{BF}(6)$ is illustrated in the following figure, where dominating set has all vertices from levels $\mathrm{L}_{0}, \mathrm{~L}_{2}$ and $\mathrm{L}_{4}$.

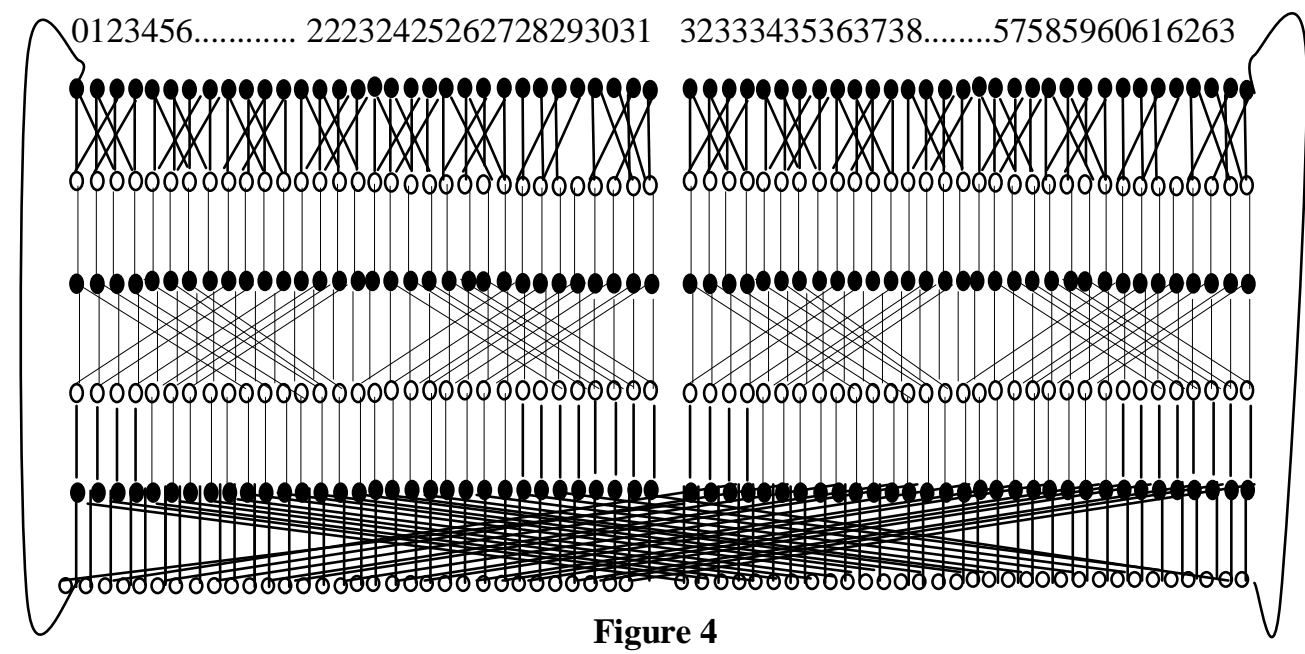

Corollary 2: $\gamma_{3}(\mathrm{BF}(\mathrm{n}))=\left\lceil\frac{n}{2}\right\rceil \mathbf{2}^{n}$ for $\mathrm{n}=5 \mathrm{k}+2,5 \mathrm{k}+3$ and $5 \mathrm{k}+4$

Theorem 2 : The 4-domination number of $B F(n)$ for $n>2$ is $\gamma_{4}(B F(n))=\left\lceil\frac{n}{2}\right\rceil 2^{n}$

Proof : By definition of edges in $\mathrm{BF}(2)$, every vertex is adjacent to three vertices only with a straight and winged edge between $(0, t)$ and $(1, t)$ for $\mathrm{t}=0,1,2,3$ which are parallel edges. So for $\mathrm{BF}(2)$ 4-domination is not possible giving $\gamma_{4}(\mathrm{BF}(2))=0$.

In $\mathrm{BF}(\mathrm{n}), \mathrm{n}>2$, every vertex is of degree 4 , all the 4 vertices must be included in dominating set. As a vertex from level $\mathrm{L}_{\mathrm{r}}$ is adjacent to two vertices each from $\mathrm{L}_{\mathrm{r}-1}$ and $\mathrm{L}_{\mathrm{r}+1}$. (modulo $\mathrm{n}$ ), to 4dominate all vertices of $\mathrm{L}_{\mathrm{r}}$ all vertices from $\mathrm{L}_{\mathrm{r}-1}$ and $\mathrm{L}_{\mathrm{r}+1}$ must be included in to 4-dominating set. So $\left\lceil\frac{n}{2}\right\rceil$ levels all $2^{\mathrm{n}}$ vertices should be in to 4-dominating set which will be the minimal 4-dominating set. Therefore $\gamma_{4}(\mathrm{BF}(\mathrm{n}))=\left\lceil\frac{n}{2}\right\rceil 2^{n}$

Observations: 1. $\gamma_{3}(B F(n))=\gamma_{4}(B F(n))$ for $n \neq 3$ as every 3 -dominating set is a 4-dominating set.

2. From [8], $\gamma(\mathrm{BF}(\mathrm{n}))=\left[\frac{n}{2}\right] 2^{n-1} \quad$ for $\mathrm{n}=2,4 \mathrm{k}, 4 \mathrm{k}+1,4 \mathrm{k}+3$

$$
=\left(\left\lceil\frac{n}{2}\right\rceil+1\right) 2^{n-1} \quad \text { for } \mathrm{n}=3,4 \mathrm{k}+2
$$

From [10] $\quad \gamma_{2}(\mathrm{BF}(\mathrm{n}))=\mathrm{k} 2^{\mathrm{n}}+\mathrm{r} 2^{\mathrm{n}-1} \quad$ for $\mathrm{n}=3 \mathrm{k}+\mathrm{r}$

$\gamma_{3}(\mathrm{BF}(\mathrm{n})), \gamma_{4}(\mathrm{BF}(\mathrm{n}))$ found above : 
The following table gives domination and $\mathrm{k}$-domination number of various butterfly graphs

\begin{tabular}{|c|c|c|c|c|}
\hline Graph & $\gamma(\mathrm{BF}(\mathrm{n}))$ & $\gamma_{2}(\mathrm{BF}(\mathrm{n}))$ & $\gamma_{3}(\mathrm{BF}(\mathrm{n}))$ & $\gamma_{4}(\mathrm{BF}(\mathrm{n}))$ \\
\hline $\mathrm{BF}(2)$ & 2 & 4 & 12 & 4 \\
\hline $\mathrm{BF}(3)$ & 6 & 8 & 32 & 32 \\
\hline $\mathrm{BF}(4)$ & 16 & 24 & 96 & 96 \\
\hline $\mathrm{BF}(5)$ & 48 & 64 & 192 & 192 \\
\hline $\mathrm{BF}(6)$ & 128 & 128 & 512 & 512 \\
\hline $\mathrm{BF}(7)$ & 256 & 320 & $\left\lceil\frac{n}{2}\right\rceil 2^{n}$ & $\left\lceil\frac{n}{2}\right\rceil 2^{n}$ \\
\hline $\mathrm{BF}(\mathrm{n}) \mathrm{n}>4$ & $\left\lceil\frac{n}{2}\right\rceil 2^{n-1}$ & $(2 \mathrm{k}+\mathrm{r}) 2^{\mathrm{n}-1}$ & \\
\hline
\end{tabular}

From above table it is evident that $\gamma_{\mathrm{k}}(\mathrm{BF}(\mathrm{n}))>\gamma(\mathrm{BF}(\mathrm{n}))$ for $\mathrm{k}=2,3,4$ and $\mathrm{n} \neq 6$.

\section{REFERENCES}

[1] Barth D. and Raspaud A, Two edge - disjoint Hamiltonian Cycles in the Butterfly Graphs, Information processing letters, 51(1994), 175-179.

[2] Berge C., Theory of graphs and its Applications, Methuen, London, 1962.

[3] Berge C., Graphs and Hypergraphs, North Holland Amsterdam, 1973.

[4] Cockayne E J, Gamble B, Shephard B, An upper bound for k-domination number of a graph, Journal of Graph Theory, 2006, Vol 9 Issue 4, pp 533-534.

[5] Fink J.F., Jacobson M.S., n-Domination in Graphs, in: Alavi Y. and Schwenk A. J.(eds), Graph Theory with Applications to Algorithms and Computer Science, New York, Wiley, 1985, 283-300.

[6] Harary F., Graph Theory, Addison-Wesley, Reading, Mass, 1972.

[7] Haynes T W, Hedetniemi S T and Slater P J, Fundamental of Domination in Graphs: Advanced Topics, (Marcel Dekker, Inc, New York,

[8] Indrani P. Kelkar, Some Studies On Domination Parameters of Butterfly Graphs, Ph.D Thesis, Sri Padmavathi Mahila Visvavidyalayam, Tirupati India, 2006.

[9] O. Ore, Theory of Graphs, Amar. Maths. Soc. Colloq. Pub., 38, Probidence, 1962.

[10] Vijayajyothi P, Indrani Kelkar, B Maheswari, 2-domination number of BF(n), Chamchuri Journal of Mathematics, Vol 1, No. 1, pp 73-80, 2008

[11] Volkman Lutz, A bound on k-domination number of graph, Czekoslovak Mathematical Journal, March 2010, Vol 60, Issue 1, pp77-83 